\title{
Acute Pancreatitis in the Setting of Acute Ischemic Stroke
}

\author{
Ashkan Mowla, ${ }^{1,}$ Navdeep S. Lail, ${ }^{1}$ and Peyman Shirani ${ }^{1}$ \\ ${ }^{1}$ Stroke Program, Department of Neurology, State University of New York at Buffalo, 100 High Street, Buffalo, NY 14202, New York, USA \\ "Corresponding author: Ashkan Mowla, Stroke Program, Department of Neurology, State University of New York at Buffalo, 100 High Street, Buffalo, NY 14202. E-mail: \\ mowla_a@yahoo.com \\ Received 2016 October 10; Accepted 2016 November 08.
}

Keywords: Stroke, Atrial Fibrillation, Pancreatitis

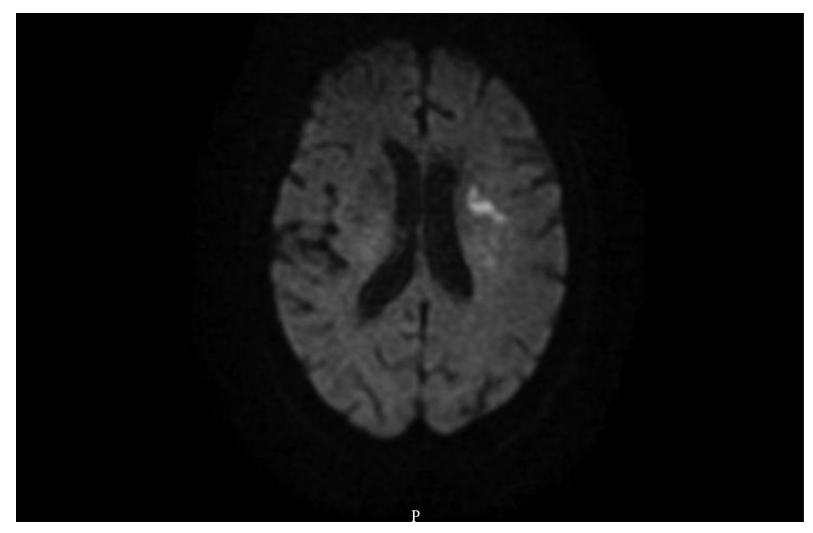

Figure 1. Brain MRI Diffusion Weighted Image Shows Diffusion Restriction in the Left Corona Radiata Consistent With Acute to Subacute Infarct

\section{Dear Editor,}

A 87- year-old lady with known history of paroxysmal atrial fibrillation (PAF) presented with sudden onset of expressive aphasia and right sided hemiparesis. She was not on any anticoagulant for her PAF at the time of presentation. She was eligible for intravenous thrombolysis (IVT) and received IVT within 3 hours of symptom onset with significant improvement in her neurological symptoms in the next 24 hours. Her Brain MRI post IVT showed an area of acute infarct in left corona radiata (Figure 1), her CT angiogram of head and neck did not show any hemodynamically significant stenosis. EKG on admission shows paroxysmal atrial fibrillation. Within 12 hours of hospital admission, she developed acute onset severe epigastric abdominal pain associated with nausea and vomiting. Serum amylase was 372 Unit/Liter (normal range: 20 - 96) and serum lipase was 364 Unit/Liter (normal range: 7 - 78). Abdomi- nal computed tomography with contrast showed acute interstitial edematous pancreatitis involving the head of the pancreas. There was no evidence of gallstone or distended gallbladder. Her serum triglyceride level and calcium were within normal range. There is no history of alcohol use or autoimmune disease.

Our patient did not have any gallstones, history of alcohol use, hypertriglyceridemia, hypercalcemia, and history of trauma or concomitant autoimmune disease which are considered the common causes of acute pancreatitis (1). Atrial fibrillation (AF) is a well-established cause of cardioembolic stroke and also peripheral embolism (2). Given the simultaneous occurrence of acute cardio-embolic ischemic stroke with acute pancreatitis in our patient, an ischemic cause for the acute pancreatitis can be assumed with a high degree of probability. Acute pancreatitis associated with PAF has been reported rarely (3) and our case is one of the first reports of acute pancreatitis possibly caused by thromboembolism associated with AF. Due to high prevalence of PAF in the elderly, it should be considered as one the potential causes of idiopathic pancreatitis particularly in the patients with evidence of simultaneous other thromboembolic events.

\section{References}

1. Namazi MR, Mowla A. Massive right-sided hemorrhagic pleural effusion due to pancreatitis; a case report. BMC Pulm Med. 2004;4:1. doi: 10.1186/1471-2466-4-1. [PubMed: 15018626].

2. Katsnelson M, Sacco RL. Stroke patients with suspected atrial fibrillation should NOT be started on anticoagulation WHILE AWAITING the results of long-term cardiac monitoring. Stroke. 2013;44(1):300-1. doi: 10.1161/STROKEAHA.112.658260. [PubMed: 23238859].

3. Janowitz P, Von Moltke A, Weidmann B. [Acute pancreatitis caused by atrial fibrillation?]. Dtsch Med Wochenschr. 2002;127(50):2669-72. doi: 10.1055/s-2002-36113. [PubMed: 12481237]. 